\title{
Multidrug resistant tuberculosis: Understanding the past for the better future
}

\section{Brahma Prakash, Dibakar Sahu', Parul Khare ${ }^{2}$,} Anuuj Kumar Bhatnagar ${ }^{3}$

Department of TB and Chest, Netaji Subhash Chandra Bose Medical College, Jabalpur, Madhya Pradesh, ${ }^{1}$ Department of TB and Chest, BPS Government Medical College for Women, Khanpur Kalan, Sonepat, Haryana, ${ }^{2}$ Department of Physiology, AlIMS, ${ }^{3}$ Department of TB and Chest, Rajan Babu Institute of Pulmonary Medicine and Tuberculosis, New Delhi, India

Address for the Correspondence: Dr. Brahma Prakash, Department of TB and Chest, Netaji Subhash Chandra Bose

Medical College, Nagpur

Road, Jabalpur - 482 003,

Madhya Pradesh, India.

E-mail: magicbrahma@rediffmail.

com

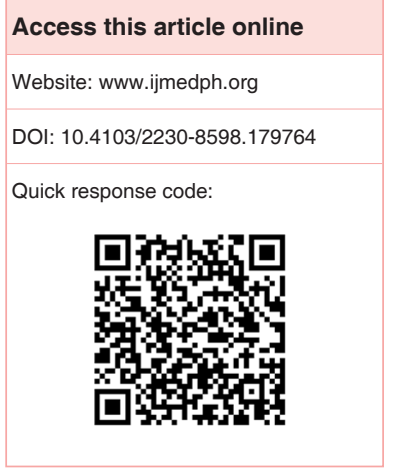

Introduction: Multidrug-resistant tuberculosis (MDR-TB) is an emerging public health problem. The study was done to evaluate the clinical profile of MDR TB patients and to study the diagnostic parameters in a cohort. Materials and Methods: All MDR-PTB patients admitted in a tertiary level TB institute for initiation of treatment for study period were enrolled. Various clinical parameters such as symptomatology profile, past treatment details, details of diagnosis, and methods of diagnosis were studied retrospectively in these patients. Results: A total of 125 patients were enrolled. An average age was $29.5 \pm 12.2$ years. The average duration of chest symptoms was $2.4 \pm 0.6$ years; cough and expectoration were the most common symptoms present in all $(100 \%)$ the patients. Two patients were treatment naïve, and 123 were retreatment cases, in which, 63 were relapse, 42 were treatment after default, and 18 were failure cases, just prior to being diagnosed as MDR-TB. Forty-three patients have received at least one of the reserve line drugs as part of anti-TB treatment in past. The average courses of treatment taken prior to been labeled as MDR-TB were 3.15 times. Majority of patients (99) had been diagnosed by using molecular-based tests. Conclusion: Use of reserve line drugs is common for treatment of TB, even without being diagnosed as MDR-TB. Both physicians- and patient-related factors responsible for the development of MDR-TB may be prevented by improving understanding of the disease and by early and proper treatment of both drugs susceptible and drug resistant TB.

Key words: Antitubercular treatment, molecular tests, multidrug-resistant tuberculosis, reserve live drugs

\section{INTRODUCTION}

Multidrug-resistant tuberculosis (MDR-TB) is becoming a major public health problem. This form of TB is not only difficult and costly to diagnose, but also tough to treat with increased morbidity and mortality as compared to the drug-susceptible TB. Mycobacterium tuberculosis is one of the important emerging pathogens. Multidrug and poly-drug resistant strains have been increasing around the world. ${ }^{[1]}$ There are 64,000 MDR-TB cases per year in India, out of which only a few are able to reach to the proper healthcare facilities to get treated. ${ }^{[2]}$ The Revised National Tuberculosis Control Program (RNTCP) under Programmatic Management of Drug Resistant Tuberculosis (PMDT) is in the phase of expansion and is making fast progress in terms of expanding the diagnostic facilities, as well as the treatment sites for such patients of $\mathrm{MDR} \mathrm{TB}^{[2]}$ but still a large proportion of $\mathrm{TB}$ patients in their early phase of illness are managed by the private health care sector by either the physician, small private hospitals and nursing homes, or corporate hospitals.

This is an open access article distributed under the terms of the Creative Commons AttributionNonCommercial-ShareAlike 3.0 License, which allows others to remix, tweak, and build upon the work non-commercially, as long as the author is credited and the new creations are licensed under the identical terms.

For reprints contact: reprints@medknow.com

How to cite this article: Prakash B, Sahu D, Khare P, Bhatnagar AK. Multidrug resistant tuberculosis: Understanding the past for the better future. Int J Med Public Health 2016;6:45-9. 
In this study, we have tried to evaluate the clinical profile of these MDR-TB patients, as well as the diagnostic parameters of the patients who were diagnosed as MDR-TB, such that an emphasis may be given by all the kind of treating facilities of TB to identify such patients early and provide or make arrangements for proper referral for these patients to get treated under PMDT, which is free of cost for the patient and therefore, cost involved in the treatment of MDR-TB patient is not a barrier for the patient.

\section{MATERIALS AND METHODS}

\section{Study design}

A retrospective case analysis of all MDR PTB cases admitted under a clinical unit of the institute, for initiation of Category IV treatment from November 2012 to April 2013 was done.

\section{Study site}

A Tertiary Care Institute for TB and pulmonary diseases at Delhi (India) under the Delhi state Government, through Municipal Corporation of Delhi, which is also a center for initiation of treatment for drug-resistant TB under PMDT and caters to inpatient needs of these patients in three out of four RNTCP districts area in Delhi. The institute has dedicated separate male and female in-patient wards for the management of drug-resistant TB, with facilities to manage these patients.

\section{Methods}

Every patient of pulmonary MDR TB who is admitted in the MDR-TB ward of the institute for initiation of Category IV treatment is first evaluated by the resident doctors of the ward, in which a detailed clinical history and examination is recorded, the past treatment records are reviewed, and the reports supporting the diagnosis of MDR-TB are attached in the case file. Then, the pretreatment evaluation with investigations is done as per the PMDT guidelines, and further initiation of treatment of MDR-TB is done under PMDT guideline ${ }^{[3]}$ by the DOTS-Plus committee.

The case files of the patients admitted between the study duration were reviewed retrospectively, and the various details of these cases were recorded on a predesigned performa with respect to various parameters such as the symptomatology profile, the past TB treatment history details, and the MDR-TB diagnosis details.

By reviewing the case history recorded in the case file, the presence of cardinal symptoms of chest disease (i.e., cough, expectoration, breathlessness, chest pain, and hemoptysis) and their duration was recorded. Patients reporting constitutional symptoms such as fever, weight loss, and loss of appetite were also recorded. History of TB and the treatment received by the patient for it was obtained from the case file. Details with respect to a number of courses of anti-TB drugs and the drugs used during these treatment, whether the only first line or also reserve line drugs received by the patient, prior to be diagnosed as MDR TB were also recorded (if they were available, in the past history segment of the history). It was assessed from their past history whether the patients were treatment naïve or retreatment cases, immediately prior to being diagnosed as MDR-TB. Further subclassification of these retreatment patients was done as per the RNTCP guideline ${ }^{[4]}$ which defines the following categories: Treatment after default, relapse, failure, and others.

In this study, the treatment aspect was not studied; hence, the parameters concerned with treatment were not recorded.

The study was approved by the Institutional Ethics Committee. Informed consent of patients was not recorded as the study was retrospective in nature, done by reviewing only the medical records of patients.

\section{Statistical analysis}

The above information from the performa was tabulated on a Microsoft Excel sheet and data were analyzed for frequencies and distribution by using statistical software SPSS version 13 (SPSS Inc., Chicago, IL, USA) on a personal computer. The level of significance was set at $P<0.05$.

\section{RESULTS}

A total of 125 patients of diagnosed MDR TB were enrolled during the study periods. Of these 125 patients, 103 were males and 22 were females.

An average age of patients was $29.5 \pm 12.2$ years ranging from 14 to 58 years, with age distribution as per male and female was $28.42 \pm 10.9$ for females and $32.42 \pm 9.2$ for males, without having significant difference according to gender $(P=0.2)$. The average weight of patient was $43.15 \pm 10.4 \mathrm{~kg}$. There was no statistically significant difference in weight found in the study in relation to duration of illness prior to diagnoses of MDR-TB.

Thirty-eight out of 125 patients had a history of smoking either presently or in past, whereas 25 patients had reported alcohol intake at least once in a week, two patients had a history of drug abuse (ganja smokers).

Average duration of chest symptoms was $2.4 \pm 0.6$ years for the patients and ranges from 3 months to 5.5 years, and the most common chest complaint was cough associated with expectoration, which was present in all the patients $(100 \%)$. The symptomatology profile of the cohort is shown in Table 1 . On reviewing the past treatment history of $\mathrm{TB}$ in these patients, it was found that all patients except two patients had a past history of anti-TB drug treatment. Two patients who were treatment naïve were suspected as MDR-TB by the treating physician of institute by sending their sputum culture at IRL as they had a molecular diagnostic test positive (XpertMDR/Rif) from a nonaccredited private laboratory.

Of these 123 patients, only five patients had taken treatment exclusively in DOTS only, prior to being diagnosed as MDR-TB 
and 21 patients had received DOTS and non-DOTS treatment both, whereas majority of patients had taken treatment from private only. The patients had been categorized as relapse, default, or failure for retreatment group by the RNTCP before sending their sputum sample for drug resistance tests. Of 123 patients, 63 were relapse, 42 were treatment after default, and 18 were failure cases.

From this past treatment history, it was found that there was a history from 43 patients who had received at least one of the second line anti-TB (reserve) drug also, along with first line antitubercular treatment (ATT), for at least 1 month in past. The most common second line AT'T prescribed to a patient by the treating doctors were quinolones, in which ofloxacin (twenty patients), levofloxacin (14 patients), and moxifloxacin (four patients) were used. The second most commonly used second-line drug was cycloserine and the third was ethionamide.

Of 125 diagnosed cases of MDR-TB, 99 patients had been diagnosed as MDR-TB by molecular diagnostic tests such as line probe assay (LPA) or GeneXpert test. The LPA and GeneXpert test are nucleic acid based tests that detect the genetic change in the Mycobacterium that is commonly responsible for resistance against isoniazid and rifampicin. LPA can detect resistance against isoniazid and rifampicin only and the GeneXpert tests detect resistance against Rifampicin only. Both these tests have been included for the diagnosis of MDR-TB under PMDT guidelines few years back. Twenty-six patients were diagnosed as MDR-TB on conventional culture (Lowenstein-Jensen [LJ] culture) or BACTEC culture and drug sensitivity testing. The LJ culture uses solid culture media, whereas the BACTEC culture uses liquid media. The drug susceptibility testing method recommended by PMDT guidelines is the proportion method, which is used by the RNTCP accredited labs for diagnosis of drug-resistant TB. In these culture methods, resistance against all the first line drugs can be checked in vitro. On the Mycobacterial culture and drug sensitivity testing, by these culture methods 16 patients had resistance to only isoniazid and rifampicin, six patients were resistant to 3 drugs, i.e. rifampicin, isoniazid, and streptomycin or ethambutol, whereas four patients were resistant to all first-line antitubercular drugs, isoniazid, rifampicin, streptomycin, and ethambutol. The details of patients drug susceptibility test (DST) results are shown in Table 2 .

\section{DISCUSSION}

MDR-TB is defined as resistance to isoniazid and rifampicin along with or without resistance to other first-line antitubercular drugs such as streptomycin, ethambutol, or pyrazinamide. ${ }^{[3]}$

The prevalence of MDR-TB is $2.2 \%$ in new patients and $15 \%$ in retreatment group. ${ }^{[2]}$ The TB prevalence may be increased in patients who are smokers. The default rates of treatment are more in cases of alcoholics. ${ }^{[5]}$

Various studies identifying roles of co-morbidities that may have a significant impact on the development of MDR-TB have reported HIV, drug abuse, and diabetes mellitus as the main causes. ${ }^{[6-10]}$

\begin{tabular}{lc}
$\begin{array}{l}\text { Table 1: Symptoms profile of multidrug resistant } \\
\text { tuberculosis patients }\end{array}$ \\
\hline Symptom & Total $\boldsymbol{n = 1 2 5 ( \% )}$ \\
\hline Cough & $125(100)$ \\
Expectoration & $125(100)$ \\
Fever & $125(100)$ \\
Breathlessness & $56(44.8)$ \\
Hemoptysis & $23(18.4)$ \\
Chest pain & $15(12)$ \\
Weight loss & $112(89.6)$ \\
Anorexia & $116(92.8)$ \\
\hline
\end{tabular}

Table 2: Resistance profile among multidrug-resistant tuberculosis patients

\begin{tabular}{lc}
\hline Resistance profile & $\boldsymbol{n = 1 2 5}(\%)$ \\
\hline $\begin{array}{l}\text { Only rifampicin resistance } \\
\text { (in molecular test based report) }\end{array}$ & $99(79.2)$ \\
$\begin{array}{l}\text { Both isoniazid and rifampicin } \\
\text { resistant only (by culture and DST) }\end{array}$ & $16(12.8)$ \\
$\begin{array}{l}\text { Three drugs resistance (H + R + } \\
\text { either S or E) (by culture and DST) }\end{array}$ & $6(4.8)$ \\
$\begin{array}{l}\text { All four drug resistant (i.e., H + R } \\
+ \text { S + E) (by culture and DST) }\end{array}$ & $4(3.2)$ \\
\hline
\end{tabular}

$\mathrm{H}=$ Isoniazid, $\mathrm{R}=$ Rifampicin, $\mathrm{S}=$ Streptomycin, $\mathrm{E}=$ Ethambutol, DST = Drugsusceptibility test

In our study also two patients were treatment naïve and 123 were of group who has received antituberculous treatment for at least 1 month in past.

The retreatment group was further comprised 63 relapse patients, 42 treatment after default, and 18 failure cases. In our study, most common group under retreatment group relapse, although 71 patients had taken the full course (either Category I or Category II regimen) of antitubercular drugs at least once in past.

Being a tertiary referral hospital with specialized care facilities as well as the main site of treatment under PMDT, most of the patients were the referral patients from various TB district TB officer, for the initiation of Category IV (DOTS-Plus) treatment and hence were now being brought under the PMDT programme, irrespective of their past history of treatment from any sources, i.e., under RNTCP or from private non-DOTS.

MDR-TB is mainly a human-made disease and three main conditions reviewed to favor the emergence and increase in MDR-TB cases are: The poor implementation of the DOTS strategy, the shortage or the poor quality of the anti-TB drugs, and the poor therapeutic adherence of the patients to the prescribed regimens. ${ }^{[1]}$ If we observe the patient related factors, it is found that forty-two patients had at least once defaulted from the treatment and adherence was an important issue with as much as 78 out of 125 patients giving a history of missing few doses and hence were irregular on treatment. There are more chances of developing drug-resistant TB in patients failing on first-line drugs than the patients of relapse group under the retreatment category. ${ }^{[12]}$ In a study, many of the patients with 
MDR-TB were defaulters at the start of their Category II regimen and financial crunch was found as a main cause of their default. In them, the mean number of AT'T courses taken before the start of Category IV was 2.85. ${ }^{[13]}$ In our study also mean number ATT courses taken by the patients is 3.15 times.

Prescription of drug-susceptible TB has to be proper in relation to the dose, duration, and regimen. It has been observed in studies that if a patient is not under DOTS, there are more chances of improper prescription, as a very few percentage of doctors might be knowing the correct dosing and treatment. In a meta-analysis done to review, the inappropriate TB treatment regimen, found that inappropriate regimens were prescribed in $67 \%$ of studies when compared with regimen recommended by the World Health Organization. ${ }^{[14]}$ The use of second-line drug should be based only on the basis of being patients diagnosed as MDR-TB on culture and DST or molecular diagnostic test for MDR-TB, but it has been observed that non-TB specialists may prescribe few of the second line antitubercular drugs in their patients either in hope of quick response or for delay in expected response, even without properly evaluating the reason for the less than expected response. In such patients who are failing on first line antitubercular drugs have more chances of being resistant to first-line drugs. ${ }^{[15]}$ The treatment of TB with the reserve line antitubercular drugs carries significantly more side effects and is usually advised for long periods as these reserve line drugs are known to be less efficacious as compared to the first-line anti-TB drugs.

Fluoroquinolones are broad-spectrum antibiotics and are used in various types of infective conditions such as bacterial respiratory tract infections, enteric fever, and other gastrointestinal infections. They have a potent antitubercular action also. Fluoroquinolone (i.e., ofloxacin, levofloxacin, and moxifloxacin) are the most commonly misused reserve line antitubercular drugs. It has been found in a study that if fluoroquinolone is used even for few days in patients has been found as a surrogate marker of MDR-TB. ${ }^{[16]}$ In a study, resistance to at least one of the second-line drug in MDR-TB patients was found as high as $44 \%$, at the start of MDR-TB treatment itself, which might be due to rampant addition of reserve line drugs to the regimens of first-line antitubercular drugs in patient with hope of quick response. ${ }^{[17]}$ Considering this nonjudicious use of reserve line drugs by the treating doctors, the authors of the study had recommended scaling up the DST facility of second line drugs and also advocated for individualized treatment regimen based on DST pattern, which might be seen as the ideal scenario for treating MDR-TB patients but may be quite difficult to achieve due to such a high number of MDR-TB patients in India and lack of adequate knowledge about the diagnosis and prescription of MDR-TB, as was found in a study, in which only six out of 106 doctors were able to write down correct prescription of MDR-TB in terms of regimen, duration, and dosage. ${ }^{[18]}$

Here, we can take an opportunity to emphasize that drug-susceptible TB may be treated using first line AT'T by either Category I treatment, i.e. for treatment naïve patients by starting with rifampicin, isoniazid, pyrazinamide, and ethambutol for 2 months and then at least rifampicin and isoniazid for 4 months and Category II or retreatment group with five drugs streptomycin, rifampicin, isoniazid, ethambutol, and pyrazinamide for 2 months, then rifampicin, isoniazid, ethambutol and pyrazinamide for 1 month, and rifampicin, isoniazid, ethambutol for next 5 months. ${ }^{[4]}$

Drug-resistant TB can be diagnosed by any of the three methods. They are the gold standard LJ culture, liquid culture (Mycobacterium Growth Indicator Tube) or molecular genotype test such as LPA or cartridge based nucleic acid amplification test or GeneXpert test. ${ }^{[3]}$ The culture-based methods are considered the gold standard for detecting MDR-TB, but few years back the molecular genotype-based diagnostic methods were also added to armamentarium for MDR-TB diagnosis by PMDT guidelines. These tests have also been approved by the world health organization for the diagnosis of MDR-TB. The early detection of MDR-TB is essential with the best appropriate measures. The genotypic methods allow for faster availability of results and have been found suitable in the clinical setting. ${ }^{[19]}$

Therefore, to strengthen the fight with the MDR-TB, it is essential to have early case detection which can be achieved by improved laboratory facilities for detection of drug resistance, the emphasis should be given for appropriate regimen being administered to the patients of both drug susceptible and drug resistant TB, which can be done by improving the knowledge about TB and imparting the education with special focus on diagnosis and treatment of TB to medical students and practicing doctors. The patient adherence to improve the cure rates has to be improved with regular health counseling and establishing proper DOTS therapy. It can be reiterated here that MDR TB should be prevented from developing, rather than managing and treating it later, by properly managing drug-susceptible TB patients. Hence, the proverb "Prevention is better than cure" stand absolutely justifiable in the case of MDR-TB as a public health problem.

\section{Financial support and sponsorship}

Nil.

\section{Conflicts of interest}

There are no conflicts of interest.

\section{REFERENCES}

1. Senol G, Komurcuoglu B, Komurcuoglu A. Drug resistance of Mycobacterium tuberculosis in Western Turkey: A retrospective study from 1100-bed teaching hospital. J Infect 2005;50:306-11.

2. Central TB Division, Ministry of Health and Family Welfare, Nirman Bhavan, New Delhi TB INDIA 2014 Revised National Tuberculosis Control Programme Annual Status Report, CTD, New Delhi, March; 2014.

3. Central TB Division, Ministry of Health and Family Welfare, Nirman Bhavan, New Delhi. Revised National Tuberculosis Control Guidelines on Programmatic Management of Drug Resistant TB (PMDT) in India; 2012.

4. Central TB Division, Ministry of Health and Family Welfare, Nirman Bhavan, New Delhi. Revised National Tuberculosis Control Programme 
Guidelines on Managing the RNTCP in Your Area - A Training Course (Module1-4), April; 2011.

5. Reis-Santos B, Gomes T, Locatelli R, de Oliveira ER, Sanchez MN, Horta BL, et al. Treatment outcomes in tuberculosis patients with diabetes: A polytomous analysis using Brazilian surveillance system. PLoS One 2014;9:e100082.

6. Marks SM, Flood J, Seaworth B, Hirsch-Moverman Y, Armstrong L, Mase S, et al. Treatment practices, outcomes, and costs of multidrug-resistant and extensively drug-resistant tuberculosis, United States, 2005-2007. Emerg Infect Dis 2014;20:812-21.

7. Schluger NW, El-Bassel N, Hermosilla $S$, Terlikbayeva A, Darisheva M, Aifah A, et al. Tuberculosis, drug use and HIV infection in Central Asia: An urgent need for attention. Drug Alcohol Depend 2013;132 Suppl 1:S32-6.

8. Prach LM, Pascopella L, Barry PM, Flood J, Porco TC, Hopewell PC, et al. Rifampin monoresistant tuberculosis and HIV comorbidity in California, 1993-2008: A retrospective cohort study. AIDS 2013;27:2615-22.

9. Tang S, Tan S, Yao L, Li F, Li L, Guo X, et al. Risk factors for poor treatment outcomes in patients with MDR-TB and XDR-TB in China: Retrospective multi-center investigation. PLoS One 2013;8:e82943.

10. Kang YA, Kim SY, Jo KW, Kim HJ, Park SK, Kim TH, et al. Impact of diabetes on treatment outcomes and long-term survival in multidrug-resistant tuberculosis. Respiration 2013;86:472-8.

11. Sotgiu G, Migliori GB. Facing multi-drug resistant tuberculosis. Pulm Pharmacol Ther 2015;32:144-8.
12. Kouamé-N'Takpé N, Horo K, Koné A, N'guessan KR, Touré K, Kouadio C et al. Clinical, microbiological and evolutionary profile of patients experiencing failures and relapses of tuberculosis in Ivory Coast. Rev Pneumol Clin 2015;71:20-6.

13. Bhatt $G$, Vyas S, Trivedil K. An epidemiological study of multi drug resistant tuberculosis cases registered under revised national tuberculosis control programme of Ahmedabad city. Indian J Tuberc 2012;59:18-27.

14. Langendam MW, van der Werf MJ, Huitric E, Manissero D. Prevalence of inappropriate tuberculosis treatment regimens: A systematic review. Eur Respir J 2012;39:1012-20.

15. Becerra MC, Freeman J, Bayona J, Shin SS, Kim JY, Furin JJ, et al. Using treatment failure under effective directly observed short-course chemotherapy programs to identify patients with multidrug-resistant tuberculosis. Int J Tuberc Lung Dis 2000;4:108-14.

16. Deutschendorf C, Goldani LZ, Santos RP. Previous use of quinolones: A surrogate marker for first line anti-tuberculosis drugs resistance in HIV-infected patients? Braz J Infect Dis 2012;16:142-5.

17. Dholakia YN, Shah DP. Clinical profile and treatment outcomes of drug-resistant tuberculosis before directly observed treatment strategy plus: Lessons for the program. Lung India 2013;30:316-20.

18. Udwadia ZF, Pinto LM, Uplekar MW. Tuberculosis management by private practitioners in Mumbai, India: Has anything changed in two decades? PLoS One 2010;5:e12023.

19. Costeira J, Pina J. Multi-drug resistant tuberculosis and the red queen - Diagnosis speed is crucial. Rev Port Pneumol 2007;13:869-77. 